\title{
Primeiro registro de escorpionismo causado por Tityus adrianoi Lourenço (Scorpiones: Buthidae)
}

\author{
First record of scorpionism caused by Tityus adrianoi \\ Lourenço (Scorpiones: Buthidae)
}

\author{
Éder Sandro Soares Álvaresi, ${ }^{1,}$ Mário De Maria ${ }^{3}$, \\ Frederico Figueiredo Amâncio ${ }^{4}$ e Délio Campolina ${ }^{4}$
}

\begin{abstract}
RESUMO
Apresentamos três casos de escorpionismo causados por Tityus adrianoi Lourenço ocorridos em Belo Horizonte e Curvelo, Minas Gerais, Brasil. $O$ atendimento aos pacientes ocorreu no mesmo dia do acidente e em todos os casos foi relatada apenas dor local, que variou de leve a intensa, sem complicações posteriores. Os três acidentes foram classificados como leves.
\end{abstract}

Palavras-chaves: Escorpionismo. Belo Horizonte. Curvelo. Minas Gerais. Tityus adrianoi.

\begin{abstract}
Three cases of scorpionism caused by Tityus adrianoi Lourenço occurring in the cities of Belo Horizonte and Curvelo, Minas Gerais State, Brazil, are presented. The patients were treated on the same day as the accident and in all cases, only local pain was reported, which varied from low to intense, without posterior complications. All three cases were classified as mild.
\end{abstract}

Key-words: Scorpionism. Belo Horizonte. Curvelo. Minas Gerais. Tityus adrianoi.

O escorpionismo no Brasil constitui um sério problema de saúde pública devido à alta toxicidade do veneno de algumas das espécies ocorrentes no país e às enormes populações destes aracnídeos encontradas em ambientes urbanizados. Entre os anos de 1990 e 1993, por exemplo, foram notificados 24.826 acidentes, com 143 óbitos $^{12}$, sendo cerca de 55\% registrados no Estado de Minas Gerais ${ }^{2}$.

No Brasil, são conhecidas mais de 90 espécies de escorpiões ${ }^{5}$. Entre estas espécies, o Tityus serrulatus, conhecido como escorpião amarelo, é o principal responsável pela maioria dos acidentes fatais registrados em Minas Gerais.

o Tityus bahiensis (Perty), chamado de escorpiãomarrom, apresenta veneno menos tóxico e é responsável por grande parte dos acidentes escorpiônicos registrados em São Paulo ${ }^{4}$. No nordeste do país, Tityus stigmurus (Thorell) responde pela maioria dos acidentes, alguns graves ${ }^{3}$. Tityus metuendus Pocock e Tityus paraensis Kraepelin, 1896 são responsabilizados por alguns acidentes no norte do país, com alguns casos isolados fatais ${ }^{5}$. As espécies Tityus charreyroni Vellard e Tityus fasciolatus Pessôa, no Distrito Federal e Goiás, podem também causar acidentes de certa gravidade ${ }^{7}$. Tityus brazilae Lourenço \& Eickstedt e Tityus neglectus Mello-Leitão, no nordeste, e Tityus mattogrossensis Borelli, no centro-oeste, norte de Minas e Bahia, são responsabilizados por acidentes de pequena gravidade, especialmente na Bahia ${ }^{8}$. Tityus trivitattus Kraepelin e Tityus costatus (Karsch) são também responsáveis por acidentes humanos moderados, mas de menor importância, devido ao baixo número de casos registrados 5 . Os acidentes com as demais espécies de Tityus são pouco conhecidos. Além deste gênero, já foram registrados no Brasil acidentes esporádicos com algumas espécies dos gêneros Bothriurus Peters, Rhopalurus Thorell e Brotheas C.L. Koch, nenhum grave5.

Tityus adrianoi Lourenço foi descrito recentemente, com base em um exemplar fêmea coletado na década de 1960 na Serra do Cipó, Minas Gerais. Este constituía o único exemplar conhecido de T. adrianoi ${ }^{6}$. Recentemente, exemplares desta

\footnotetext{
1. Departamento de Zoologia do Instituto de Biociências da Universidade de São Paulo, São Paulo, SP. 2. Laboratório de Artrópodes do Instituto Butantan. São Paulo, SP. 3. Laboratório de Aracnologia do Departamento de Zoologia do Instituto de Ciências Biológicas da Universidade Federal de Minas Gerais, Belo Horizonte, MG. 4. Serviço de Toxicologia de Minas Gerais do Hospital João XXIII, Belo Horizonte, MG.

Endereço para correspondência: Prof. Mário De Maria. Laboratório de Aracnologia/Departamento de Zoologia/ICB/UFMG. Av. Antonio Carlos 6627, 31270-901 Belo Horizonte, MG.

Tel: $55113499-2916$

e-mail:demaria@icb.ufmg

Recebido para publicação em 11/4/2006

Aceito em 28/6/2006
} 
espécie foram coletados na região metropolitana de Belo Horizonte, Minas Gerais (Álvares ESS, De Maria M: dados não publicados). Apresentamos neste trabalho, o relato dos três primeiros casos de envenenamento atribuídos a $T$. adrianoi.

\section{RELATO DOS CASOS}

Caso 1. Paciente de 26 anos foi recebido às 10:30am do dia 08/07/2004, vítima de um acidente com um escorpião no Bairro Santa Lúcia, Belo Horizonte, às 09:50am do mesmo dia. A ferroada ocorreu no terço medial da coxa esquerda, sendo relatada pelo paciente dor intensa no local, logo após o acidente. Não foram observados outros sintomas. 0 tratamento foi efetuado apenas com a administração de $2 \mathrm{ml}$ de dipirona endovenosa para alívio da dor.

Caso 2. Criança do sexo masculino de 11 anos, atendida às 7:00am do dia 15/09/2004, foi ferroada no quinto pododáctilo. 0 acidente ocorreu no mesmo dia do atendimento, na casa do paciente, no Bairro Taquaril, por volta das 6:00am. Foi registrada apenas dor local. 0 tratamento incluiu analgesia com dipirona em gotas. 0 paciente foi liberado 12 horas após o acidente.

Caso 3. Paciente do sexo masculino, 51 anos, foi ferroado na mão esquerda. 0 acidente ocorreu na Cidade de Curvelo, $160 \mathrm{~km}$ ao norte de Belo Horizonte, no dia 18/10/2004 às 15:40pm, e o atendimento foi no mesmo dia, às 19:00, no Hospital João XXIII. Foi registrada dor local leve, com parestesia no membro superior esquerdo, sem necessidade de analgesia. 0 paciente foi liberado 6 horas após o acidente.

\section{DISCUSSÃo}

Nos três casos, o exemplar do escorpião responsável pela ferroada foi capturado logo após o acidente e levado pelos pacientes ao Serviço de Toxicologia de Minas Gerais, no Hospital João XXIII. Os espécimens foram identificados como T. adrianoi, através de comparações com a descrição original ${ }^{6}$. Os exemplares encontram-se depositados na coleção LAMG (Laboratório de Aracnologia, Departamento de Zoologia, Instituto de Ciências Biológicas da Universidade Federal de Minas Gerais); IBSP (Instituto Butantan, São Paulo) e na coleção de referência do Serviço de Toxicologia do Hospital João XXIII, FHEMIG.

A dor relatada no local da ferroada pelos pacientes logo após o acidente é comum no escorpionismo, estando presente em quase a totalidade dos $\operatorname{casos}^{12}$. 0 registro apenas de dor local com parestesia no membro superior esquerdo em apenas um dos casos, levou à classificação destes acidentes como leve. Cerca de $95 \%$ dos casos de escorpionismo ocorridos em Belo Horizonte são classificados como leves e não requerem medidas de tratamento específico, como a administração de soro antiescorpiônico. Os acidentes descritos nos três casos apresentaram-se clinicamente indistinguíveis de acidentes leves causados por T. serrulatus ou T. bahiensis. Este fato, possivelmente, contribui para identificação errônea da espécie causadora do acidente levando-se em conta apenas a sintomatologia.

Os hábitos e preferências ecológicas de Tityus adrianoi ainda não são conhecidos, mas os exemplares até então coletados foram encontrados em ambientes naturais ou em bairros próximos a remanescentes de vegetação nativa na Cidade de Belo Horizonte. Possivelmente, esta espécie possui necessidades mais restritas de micro-hábitats, não invadindo ambientes domiciliares com tanta facilidade quanto $T$. serrulatus ou $T$. bahiensis.

\section{AGRADECIMENTOS}

Agradecemos a Sylvia Lucas pela leitura do manuscrito e sugestões e a Denise Candido pelo auxílio na identificação dos escorpiões. Ao corpo clínico do Serviço de Toxicologia do Hospital João XXIII, somos gratos pelas informações acrescentadas às fichas dos pacientes.

\section{REFERÊNCIAS BIBLIOGRÁFICAS}

1. Cupo P, Azevedo-Marques MM, Hering SE. Escopionismo. In: Cardoso JCL, França FOS, Wen FH, Málaque CMS, Haddar JR (eds) Animais Peçonhentos no Brasil: biologia, clínica e terapêutica dos acidentes, Editora Sarvier, São Paulo, p. 198-208, 2003.

2. Cupo P, Jurca M, Azevedo-Marques MM, Oliveira JS, Hering SE. Severe scorpion envenomation in Brazil. Clinical, laboratory and anatomopathological aspects. Revista do Instituto de Medicina Tropical de São Paulo 36: 67-76, 1994.

3. Eickstedet VR. Escorpionismo por Tityus stigmurus no Nordeste do Brasil (Scorpiones, Buthidae). Memórias do Instituto Butantan 47-48: 133-137, 1983

4. Eickstedet VR, Ribeiro LA, Candido DM. Evolution of scorpionism by Tityus baihensis (Perty) and T. serrulatus Lutz and Mello and geographical distribution of the two species in the state of São Paulo, Brazil Journal of Venom and Animal Toxins 2: 92-105, 1996.

5. Lourenço WR. Scorpions of Brazil. Les Éditions l'. If. Paris, France, 2002.

6. Lourenço WR. Description of a new species of Tityus (Scorpiones, Buthidae) from Serra do Cipó in the State of Minas Gerais, Brazil. Revue Suisse de Zoologie 110: 427-435, 2003.

7. Lourenço WR, Knox MB, Yoshizawa MAC. L'invasion d'une communauté à le stade initial d'une succession secondaire par une espèce parthénogénétique de scorpion. Biogeographica 70: 77-91, 1994.

8. Silva RML, Amorim AM, Brazil TK. Scorpions of medical importance in Bahia, Brazil. Journal of Venom and Animal Toxins 3: 250, 1997.

9. Soares MRM, Azevedo CS, De Maria M. Escorpionismo em Belo Horizonte, MG: um estudo retrospectivo. Revista da Sociedade Brasileira de Medicina Tropical 35: 359-363, 2002. 